\title{
No Association of Chronic Cerebrospinal Venous Insufficiency with Multiple Sclerosis
}

\author{
Robert J Fox, Claudiu Diaconu, Leasa Baus, Alia Grattan, Irene Katzan, Jar-Chi Lee, Larry Raber, \\ Alexander Rae-Grant, Sneha Ramesh, Mei Lu, Esther SH Kim
}

Keywords: Multiple Sclerosis, Chronic Cerebrospinal Venous Insufficiency

doi:10.1017/cjn.2015.245

Can J Neurol Sci. 2016; 43: 195-197

Chronic cerebrospinal venous insufficiency (CCSVI) has been described as a venous condition with abnormal flow in extracranial and intracranial veins. ${ }^{1}$ CCSVI, as evaluated by ultrasound, has been hypothesized to be associated with multiple sclerosis (MS), possibly even causing MS. ${ }^{1}$ Five venous characteristics (often referred to as criteria) were recommended to evaluate CCSVI: criterion 1 evaluates extracranial reflux in the internal jugular or vertebral veins in sitting and supine positions; criterion 2 assesses reflux in the deep cerebral veins; criterion 3 assesses proximal internal jugular vein stenosis; criterion 4 assesses lack of flow in the internal jugular or vertebral veins in sitting and supine positions; and criterion 5 assesses reverted postural control of the main cerebral venous outflow pathway. ${ }^{1}$

Subsequent to the initial reports by Zamboni et al, prevalence studies of CCSVI in MS have reported conflicting results. ${ }^{2-4}$ Importantly, some studies have found a very low prevalence of CCSVI, ${ }^{5-8}$ suggesting that the ultrasound assessments in those studies were conducted very differently from the original reports. ${ }^{9}$ We assessed the prevalence of CCSVI in both MS and non-MS populations, utilizing standardized ultrasound methodology and blinded evaluators.

\section{MethodS}

Using an Institutional Review Board-approved protocol, MS and non-MS controls were evaluated for CCSVI. Subjects with a definite diagnosis of MS, aged 21- 65 years were invited to participate in this study at the Mellen Center, Cleveland Clinic, Cleveland, Ohio, USA. Age matched healthy controls were recruited from the caregiver population and controls with other neuro-inflammatory disorders were recruited from neurologic clinics at the Cleveland Clinic. All ultrasounds were conducted using a Biosound MyLab25Gold, which included traditional trans-cranial Doppler (TCD) and Quality Doppler Profiles (QDP) technology. The Biosound ultrasound machine is capable of high resolution B-mode, which is used to evaluate stenoses in proximal internal jugular veins, (criterion 3).The Biosound's QDP technology is specifically used to assess reflux in the deep cerebral veins (criterion 2). ${ }^{1}$ Prior to the study, two sonographers obtained formal training in CCSVI assessments using the BioSound machine, ${ }^{10}$ and ultrasound methodology was further standardized to reduce ambiguity. All sonographers hold Registered Vascular Technologist (RVT) credentials. Sonographers were blinded to diagnosis, including the use of separate research staff to position the subject prior to the arrival of the sonographer.
The internal jugular, vertebral, and deep cerebral veins were assessed and measured in both supine and sitting positions. Based upon previous publications, two interpretations ${ }^{1,2}$ of CCSVI criteria were utilized: Narrow Criteria, which did not include either B-mode intraluminal abnormalities (criterion 3) or QDP technology for deep vein reflux (criterion 2); and Broad Criteria, which included both of these. ${ }^{11}$ The Broad Criteria, in fact, have been recently recommended by the International Society of NeuroVascular Disease for CCSVI diagnosis. ${ }^{12}$ All ultrasounds were over-read by a Registered Physician in Vascular Interpretation (EK or ML), who was also blinded to MS diagnosis.

Based upon the original Zamboni report of $100 \%$ sensitivity and $100 \%$ specificity of CCSVI, sample size was estimated at $\mathrm{n}=15$ per group to replicate those observations. We increased the original sample size to 120 ( $80 \mathrm{MS}$ and 40 non-MS controls) to have a $90 \%$ power to detect an absolute difference of $35 \%$ in diagnostic classification (i.e. $30 \%$ positive in controls and $65 \%$ positive in MS subjects; Chi-square with continuity correction, $\alpha=0.05$, 2-tailed). Based upon several reports finding no association of CCSVI with MS, an interim futility analysis was conducted after 61 of the anticipated 120 subjects were enrolled. That interim analysis found that further enrollment was unlikely to alter the group differences observed to date.

\section{RESULTS}

We enrolled 61 MS subjects (4 CIS, 28 RRMS, 19 SPMS and 10 PPMS; 42 females; mean age $53.84 \pm 9.51$ years) and 20 non-MS controls (15 healthy subjects and 5 with other neurological diseases; 10 females; mean age $52.21 \pm 11.25$ years).

The incidence of subjects meeting CCSVI criteria was determined using two interpretations of the ultrasound assessments, which differed in the way criteria 2 and 3 were analyzed. For criterion 2, the narrow interpretation used TCD to assess deep cerebral vein reflux whereas the broad interpretation used QDP. For criterion 3 , the narrow interpretation used only B-mode

From the Cleveland Clinic Lerner College of Medicine of Case Western Reserve University (CD); Mellen Center for Multiple Sclerosis (RJF, AR-G, SR); Cerebrovascular Center (ML); Neurological Institute; Neurovascular Laboratory (LB, IK, LR); Imaging Institute: Heart and Vascular Institute (AG, ESHK); Department of Quantitative Health Sciences (J-CL), Cleveland Clinic, Cleveland, OH, USA

Received OCtober 30, 2014. Final Revisions Submitted April 27, 2015. Correspondence to: Robert J Fox, Mellen Center for Multiple Sclerosis, Cleveland Clinic, 9500 Euclid Avenue, U10, Cleveland, OH 44120, USA. Email: foxr@ccf.org 

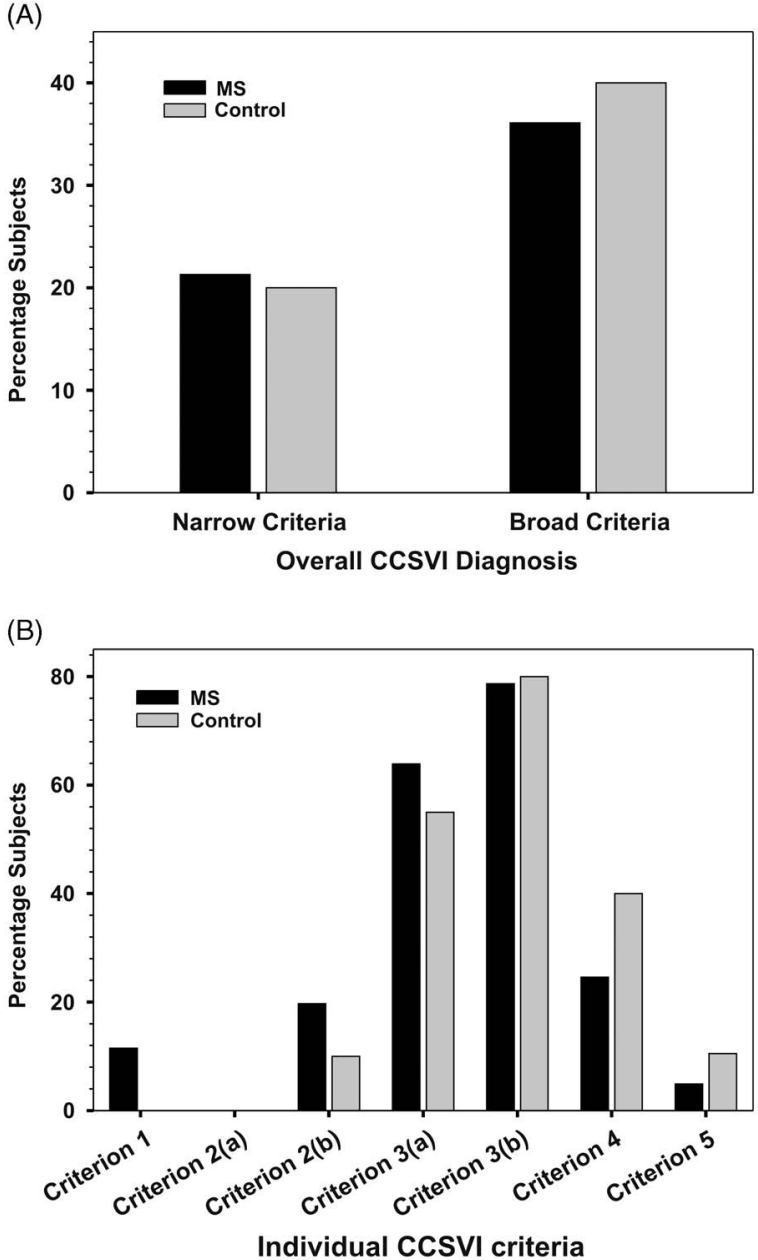

Figure 1: Percentage of Subjects meeting CCSVI criteria. (A) Percentage of subjects meeting overall CCSVI diagnosis using narrow and broad criteria. (B) Percentage of subjects meeting individual CCSVI criterion, both narrow $(n)$ and broad $(b)$ interpretations.

luminal stenosis $\left(<0.30 \mathrm{~cm}^{2}\right)$ as a measure whereas, the broad interpretation included other B-mode abnormalities (e.g. flap or septum) as well.

Using Narrow Criteria, the incidence of subjects meeting two or more CCSVI criteria was $20.0 \%$ in controls and $21.3 \%$ in MS (Fisher exact test, $\mathrm{p}=0.99$; Figure 1A). Using Broad Criteria, the incidence of subjects meeting two or more CCSVI was $40 \%$ in controls and $36.1 \%$ in MS (Chi-square test, $\mathrm{p}=0.75$; Figure $1 \mathrm{~A}$ ). Using either criteria, there was no significant difference in CCSVI between MS and controls. In both MS and control groups, there was almost a doubling of the proportion of subjects meeting overall CCSVI definition when using the Broad Criteria. Similarly, there was no significant difference in the proportion of MS and control subjects that met any of the individual ultrasound criteria (Figure 1B).

\section{Discussion}

Using blinded sonographers specifically trained in CCSVI assessments, as well as QDP technology, we observed no significant difference in prevalence of CCSVI between MS and non-MS subjects (Figure 1A). Regardless of whether the more liberal Broad Criteria or the restrictive Narrow Criteria were used, the proportion of subjects meeting two or more CCSVI criteria was the same in both MS and non-MS controls (Figure 1A).

Our study adds to the accumulating evidence of contemporary, independent studies demonstrating no association between MS and the vascular condition called CCSVI. Baretto et $\mathrm{al}^{13}$ found a CCSVI prevalence of $3.88 \%$ among $200 \mathrm{MS}$ patients and $7.14 \%$ among non-MS controls. Traboulsee et al found a CCSVI prevalence of $44 \%$ among 79 MS patients and $45 \%$ among 43 nonMS controls. ${ }^{14}$ Costello et al used both extracranial Doppler ultrasonography and magnetic resonance venography to test the CCSVI hypothesis and found no differences in CCSVI prevalence between patients and controls. ${ }^{15}$ In the largest study to date, Comi et al found a CCSVI prevalence of $3.26 \%$ among $1165 \mathrm{MS}$ patients and $2.13 \%$ among 376 non-MS controls. ${ }^{16}$

As we have reported previously, different tools to assess for CCSVI and different interpretations of CCSVI criteria can alter the proportions of subjects meeting CCSVI criteria. The original description of CCSVI utilized the new Biosound MyLab25Gold ultrasound, which utilizes Quality Doppler Profiles (QDP) technology for assessment of flow in the deep cerebral veins. Due to limited availability of the Biosound platform, many subsequent CCSVI studies have utilized TCD instead. Our study highlights the difference in sensitivity between QDP and TCD; 14/81 (17.3\%) subjects met Criterion 2 using QDP, while 0/81 (0\%) met Criterion 2 using TCD. Similarly, Criterion 3 was met in 50/81 (61.7\%) using Narrow Criteria, but 64/81 (79\%) using Broad Criteria (Figure 1B). Our observations provide one explanation for the vastly different incidence of CCSVI in healthy controls (and people with MS) among different studies. Some studies found a prevalence of $0-2 \%$ in non-MS controls, ${ }^{5-8}$ while others found a prevalence of $22.7-45 \%$ in non-MS controls. ${ }^{14,17,18}$ These dramatic differences are most certainly due to differences in how the CCSVI ultrasound assessments were conducted and interpreted.

The original protocol for ultrasound assessment of CCSVI has been modified over time. The International Society for NeuroVascular Disease published updated methodology in $2011^{19,20}$ and then again in $2014 .{ }^{12}$ The change in ultrasound assessments for CCSVI over time and the differences in ultrasound technologies (for example, trans-cranial Doppler and Quality Doppler Profiles technologies) likely contributed to the differences in results from different investigational teams. Our observations highlight the importance of standardizing assessments and their interpretations when new techniques and technologies are introduced.

Unlike the initial unblinded assessments of CCSVI, ${ }^{1,21}$ our study was conducted with careful attention to blinding. Each subject (MS and non-MS controls) was brought into the ultrasound examination room and positioned by a separate research staff, before the arrival of the sonographer. Thus, sonographers were blinded to the physical impairments of the study subjects. Each ultrasound study was then over-read at a later time by an independent, blinded physician certified in vascular interpretations. The absence of such rigorous blinding is a possible explanation for some of the previous studies that found associations of CCSVI with MS. Some studies that found a difference in CCSVI prevalence between MS and non-MS controls report having been conducted blinded, although the details of the blinding were typically not described. ${ }^{1,17}$ Blinding may explain some of the differences in results between different studies but seems not to explain all the differences. 
Other factors that may influence ultrasound assessments for CCSVI include hydration. We found that rehydration after mild, overnight dehydration can have a significant impact on CCSVI ultrasound assessment, with five of seven subjects no longer meeting CCSVI criteria following rehydration. ${ }^{22}$ People with MS often prefer to be mildly dehydrated because of neurogenic bladder dysfunction and this may have contributed to some studies finding an increased prevalence of CCSVI among people with MS.

In summary, our observations do not support an increased prevalence of CCSVI in MS and suggest against a pathogenic role of CCSVI in MS. Combined with other negative association studies of CCSVI with MS, further study of CCSVI in MS appears to be unwarranted.

\section{ACKNOWLEDGEMENTS}

Supported by the National MS Society (RC 1004-A-5).

\section{Contributions}

Dr. Fox: study concept and design, study supervision and coordination, analysis and interpretation of data, obtained funding, manuscript preparation; Dr. Diaconu: study conduct and coordination, analysis and interpretation of data, manuscript review; Ms. Baus, Ms. Grattan, Dr. Katzan and Mr. Raber: study conduct, manuscript review; Dr. Rae-Grant: study design, study supervision and coordination, interpretation of data, manuscript review.

Ms. Lee: statistical analysis, manuscript review; Dr. Ramesh: study conduct and coordination, data analysis, manuscript preparation; Dr. Lu: study supervision, analysis and interpretation of data, manuscript review; Dr. Kim: study supervision, analysis and interpretation of data, manuscript review.

\section{Disclosures}

Robert Fox has the following disclosures: Allozyne, Consultant, Consulting fees; Anavir, Consultant, Consulting fees; Biogen-Idec, Consultant and Advisory committee, Consulting fees, Novartis, Consultant and Advisory committee, Consulting fees, Questcor, Consultant, Consulting fees, Teva, Consultant, Consulting fees, Xenoport, Consultant, Consulting fees.

Esther S.H. Kim has the following disclosures: Phillips Ultrasound, Consultant, Consulting fees; GE, Researcher, Grant funding.

Claudiu Diaconu, Leasa Baus, Alia Grattan, Irene Katzan, Jar-Chi Lee, Larry Raber, Alexander Rae-Grant, Sneha Ramesh, and Mei Lu do not have anything to disclose.

\section{REFERENCES}

1. Zamboni P, Galeotti R, Menegatti E, et al. Chronic cerebrospinal venous insufficiency in patients with multiple sclerosis. J Neurol Neurosurg Psychiatry. 2009;80:392-9.

2. Zivadinov R, Marr K, Cutter G, et al. Prevalence, Sensitivity and Specificity of Chronic Cerebrospinal Venous Insufficiency in MS. Neurology. 2011;77:138-44.

3. Khan O, Tselis A. Chronic cerebrospinal venous insufficiency and multiple sclerosis: science or science fiction? J Neurol Neurosurg Psychiatry. 2011;82:355.
4. Tromba L, Blasi S, Vestri A, Kiltzanidi D, Tartaglia F, Redler A. Prevalence of chronic cerebrospinal venous insufficiency in multiple sclerosis: a blinded sonographic evaluation. Phlebology. 2015;30:52-60.

5. Doepp F, Paul F, Valdueza JM, Schmierer K, Schreiber SJ. No cerebrocervical venous congestion in patients with multiple sclerosis. Ann. Neurol. 2010;68:173-83.

6. Baracchini C, Perini P, Calabrese M, Causin F, Rinaldi F, Gallo P. No evidence of chronic cerebrospinal venous insufficiency at multiple sclerosis onset. Ann Neurol. 2011;69:90-9.

7. Van den Berg PJ, Van den Berg GB, Westerhuis LW, Visser LH. Occurrence of CCSVI in patients with MS and its relationship with iron metabolism and varicose veins. Eur $\mathrm{J}$ Neurol. 2013;20:519-26.

8. Rodger IW, Dilar D, Dwyer J, et al. Evidence against the involvement of chronic cerebrospinal venous abnormalities in multiple sclerosis. A case-control study. PLoS One. 2013;8: e72495

9. Valdueza JM, Doepp F, Schreiber SJ, et al. What went wrong? The flawed concept of cerebrospinal venous insufficiency. J Cereb Blood Flow Metab. 2013;33:657-68.

10. BNAC. Buffalo Neuroimaging Analysis Center. http://www.bnac. net/. Accessed 2 June, 2014.

11. Kim SH, Diaconu C, Fox RJ, et al. Chronic Cerebrospinal Venous Insufficiency: Pitfalls and Perils of Ultrasound Assessment. J Ultrasound Med. 2015;34:1097-106.

12. Zivadinov R, Bastianello S, Dake MD, et al. Recommendations for multimodal noninvasive and invasive screening for detection of extracranial venous abnormalities indicative of chronic cerebrospinal venous insufficiency: a position statement of the International Society for Neurovascular Disease. J Vasc Interv Radiol. 2014;25:1785-94 e1717.

13. Barreto AD, Brod SA, Bui TT, et al. Chronic cerebrospinal venous insufficiency: case-control neurosonography results. Ann Neurol. 2013;73:721-8

14. Traboulsee AL, Knox KB, Machan L, et al. Prevalence of extracranial venous narrowing on catheter venography in people with multiple sclerosis, their siblings, and unrelated healthy controls: a blinded, case-control study. Lancet. 2014; 383:138-45.

15. Costello F, Modi J, Lautner D, et al. Validity of the diagnostic criteria for chronic cerebrospinal venous insufficiency and association with multiple sclerosis. CMAJ. 2014;186:E418-26.

16. Comi G, Battaglia MA, Bertolotto A, et al. Observational case-control study of the prevalence of chronic cerebrospinal venous insufficiency in multiple sclerosis: results from the CoSMo study. Mult Scler. 2013;19:1508-17.

17. Zivadinov R, Marr K, Cutter G, et al. Prevalence, sensitivity, and specificity of chronic cerebrospinal venous insufficiency in MS. Neurology. 2011;77:138-44.

18. Leone MA, Raymkulova O, Naldi P, et al. Chronic cerebrospinal venous insufficiency is not associated with multiple sclerosis and its severity: a blind-verified study. PLoS One. 2013;8: e56031.

19. Nicolaides AN, Morovic S, Menegatti E, Viselner G, Zamboni P. Screening for chronic cerebrospinal venous insufficiency (CCSVI) using ultrasound: recommendations for a protocol. Funct Neurol. 2011;26:229-48.

20. Zamboni P, Morovic S, Menegatti E, Viselner G, Nicolaides AN. Screening for chronic cerebrospinal venous insufficiency (CCSVI) using ultrasound-recommendations for a protocol. Int Angiol. Dec 201130 571-97.

21. Al-Omari MH, Rousan LA. Internal jugular vein morphology and hemodynamics in patients with multiple sclerosis. Int Angiol. 2010;29:115-20.

22. Diaconu CI, Fox RJ, Grattan A, et al. Hydration status substantially affects chronic cerebrospinal venous insufficiency assessments. Neurol Clin Pract. 2013;3:386-91. 